\title{
Evolution of an electron-positron plasma produced by induced gravitational collapse in binary-driven hypernovae
}

\author{
J. D. Melon Fuksman ${ }^{1,2, \star}$, L. Becerra ${ }^{1,2}$, C. L. Bianco ${ }^{1,2}$, M. Karlica ${ }^{1,2,3}$, M. Kovacevic ${ }^{1,2,3}$, R. Moradi ${ }^{1,2}, M$. \\ Muccino $^{1,2}$, G. B. Pisani ${ }^{1,2}$, D. Primorac ${ }^{1,2}$, J. A. Rueda ${ }^{1,2,4}, R$. Ruffini ${ }^{1,2,3,4}$, G. V. Vereshchagin ${ }^{1,2}$, and $Y$. Wang $^{1,2}$ \\ ${ }^{1}$ Dipartimento di Fisica, Sapienza Università di Roma, Piazzale Aldo Moro 5, 00185 Rome, Italy \\ ${ }^{2}$ ICRANet, Piazza della Repubblica 10, 65122 Pescara, Italy \\ ${ }^{3}$ Université de Nice Sophia Antipolis, CEDEX 2, Grand Château Parc Valrose, Nice, France \\ ${ }^{4}$ ICRANet-Rio, Centro Brasileiro de Pesquisas Físicas, Rua Dr. Xavier Sigaud 150, 22290-180 Rio de Janeiro, Brazil
}

\begin{abstract}
The binary-driven hypernova (BdHN) model has been introduced in the past years, to explain a subfamily of gamma-ray bursts (GRBs) with energies $E_{i s o} \geq 10^{52} \mathrm{erg}$ associated with type Ic supernovae. Such BdHNe have as progenitor a tight binary system composed of a carbon-oxigen (CO) core and a neutron star undergoing an induced gravitational collapse to a black hole, triggered by the $\mathrm{CO}$ core explosion as a supernova (SN). This collapse produces an optically-thick $e^{+} e^{-}$plasma, which expands and impacts onto the $\mathrm{SN}$ ejecta. This process is here considered as a candidate for the production of X-ray flares, which are frequently observed following the prompt emission of GRBs. In this work we follow the evolution of the $e^{+} e^{-}$plasma as it interacts with the SN ejecta, by solving the equations of relativistic hydrodynamics numerically. Our results are compatible with the Lorentz factors estimated for the sources that produce the flares, of typically $\Gamma \lesssim 4$.
\end{abstract}

\section{Introduction}

The induced gravitational collapse (IGC) (see, e.g., [1], [2], [3]) has been proposed in the past years, as a way to explain a sub-class of long gamma-ray bursts (GRBs) called binary-driven hypernovae (BdHNe), characterised by an isotropic energy $E_{i s o} \geq 10^{52} \mathrm{erg}$ and a rest-frame spectral peak energy between 0.2 and $2 \mathrm{MeV}$. The model considers a binary system formed by a carbon-oxygen (CO) core and a neutron star (NS), which undergo a tight orbit. If the core-collapse of the $\mathrm{CO}$ star produces a supernova explosion, the ejected material may trigger an hypercritical accretion process onto the NS, due to a copious neutrino emission and the trapping of photons within the accretion flow. This process can cause the NS to collapse as well, thus forming a black hole (BH). It has been proposed in [4] and, e.g., [5], that such collapse can lead to the formation of an $e^{+} e^{-}$plasma, that later expands and interacts with the $\mathrm{SN}$ ejecta, finally producing a GRB.

In this scenario, the major portion of the optically thick $e^{+} e^{-}$-baryon plasma originating from the collapse expands away from the supernova ( $\mathrm{SN}$ ), giving rise to the canonical GRB prompt emission. This emission occurs at approximately $10^{15}-10^{17} \mathrm{~cm}$ from the $\mathrm{BH}$, and is measured to come from material that expands at Lorentz factors $\Gamma \sim 10^{2}-10^{3}$ (see e.g. [5]). Right after this first stage, that can last up to $\sim 100 \mathrm{~s}, X$-ray flares are frequently observed, followed by the so-called "plateau" and finally by the late decay of the X-ray afterglow [6]. By studying

\footnotetext{
^e-mail: david.melon@icranet.org
}

the time evolution of the thermal component of the X-ray flares, it can be inferred that they originate from regions which move at roughly $\Gamma \lesssim 4$, as pointed out in [6].

These differences in the features of the prompt emission and the flares can be explained in terms of the IGC model. In it, the prompt emission is produced after the interaction of the $e^{+} e^{-}$with the $\mathrm{SN}$ ejecta, in a direction that corresponds to lower overall densities along the line of sight of an external observer (see [7] and Fig. 1). On the other hand, as the binary system keeps spinning, the mass density profile along that line changes. If a bigger amount of mass gets between the $\mathrm{BH}$ and the observer (see [8] and Fig. 1), an X-ray flare is emitted at the moment the plasma escapes the outermost regions of the SN ejecta, namely, at the shock breakout. Due to the deceleration of the shock by its interaction with the surrounding material, the Lorentz factors measured for the flares will be smaller than for the prompt emission.

In this work we describe numerically the evolution of the plasma along different directions, and study the compatibility of the IGC model with some of the abovementioned observational features.

\section{Equations and numerical scheme}

We have modeled the evolution of the $e^{+} e^{-}$plasma and the SN ejecta following a single-fluid approach, where all the involved particle species, in this case baryons, photons, electrons and positrons, coming either from the plasma or the SN, are in local thermodynamic equilibrium (LTE). 

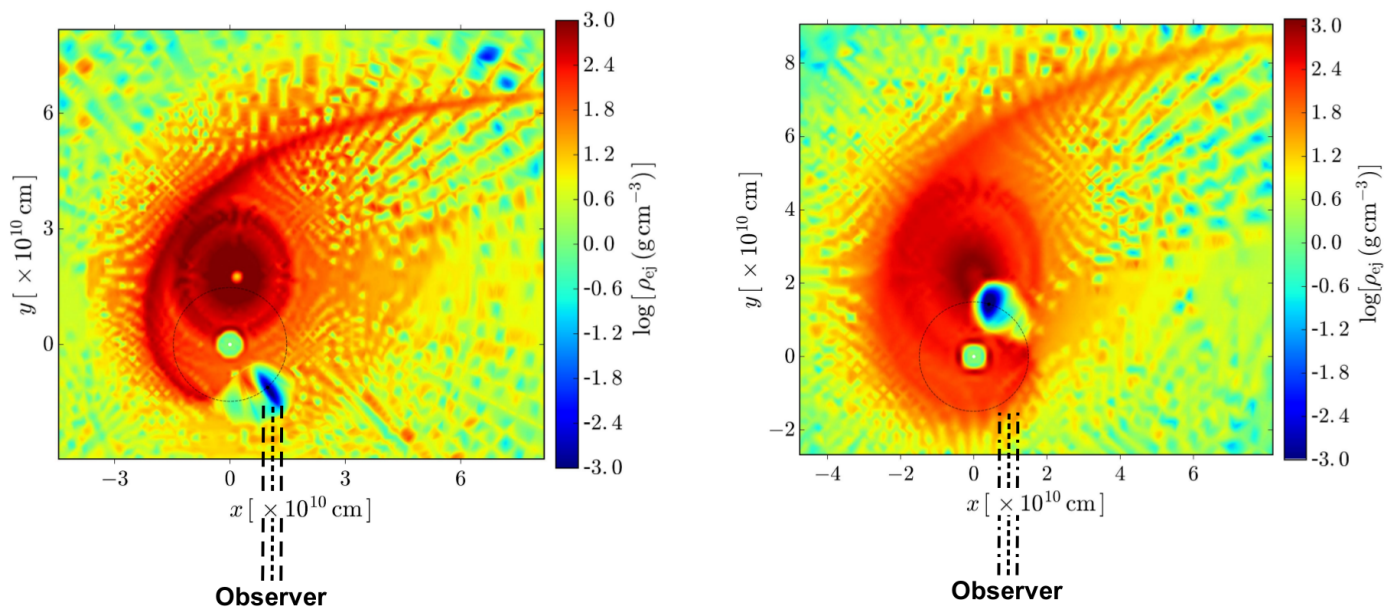

Figure 1. Density profiles calculated in [8] corresponding to the SN ejecta at (left) the moment of the collapse and (right) $100 \mathrm{~s}$ later. The line of sight of an observer that sees the initial prompt emission is indicated, to point out the change of the mass profile along it.

Under this assumption, the dynamics of the resulting fluid is governed by the equations of relativistic hydrodynamics (RHD). Throughout this work, we have numerically solved the one-dimensional RHD equations with the additional assumption of spherical symmetry, considering only a dependence of the intensive variables on the radial spherical coordinate. This allows us to study the evolution of the plasma along one selected radial direction at a time, thus to consider the different density distributions as seen through each direction. In the absence of gravity, the resulting equations of motion can be written as follows:

$$
\begin{aligned}
\frac{\partial(\rho \Gamma)}{\partial t}+\nabla \cdot(\rho \Gamma \mathbf{v}) & =0, \\
\frac{\partial m_{r}}{\partial t}+\nabla \cdot\left(m_{r} \mathbf{v}\right)+\frac{\partial p}{\partial r} & =0, \\
\frac{\partial \mathcal{E}}{\partial t}+\nabla \cdot(\mathbf{m}-\rho \Gamma \mathbf{v}) & =0,
\end{aligned}
$$

where $\rho$ is the comoving mass density, $\mathbf{v}$ is the fluid velocity (natural units where $c=1$ are used), $\Gamma=\left(1-\mathbf{v}^{2}\right)^{-\frac{1}{2}}$ is the Lorentz factor, $\mathbf{m}$ the total momentum density, and the subscript $r$ indicates the radial component of a vector. The momentum density $\mathbf{m}$ is defined as $\mathbf{m}=h \Gamma^{2} \mathbf{v}$, where $h$ is the comoving enthalpy density, given by $h=\rho+\epsilon+p$, where $p$ is the gas pressure and $\epsilon$ its internal energy density, both measured in the comoving frame. Finally, we denote by $\mathcal{E}$ the value of $\epsilon$ in the laboratory (or coordinate) frame. In the above formulation of these equations, we compute this energy density by substracting the coordinate mass density $\rho \Gamma$ to the $(0,0)$ component of the fluid's energy-momentum tensor $T^{\mu \nu}$, as

$$
\begin{aligned}
\mathcal{E} & =T^{00}-\rho \Gamma \\
& =h \Gamma^{2}-p-\rho \Gamma .
\end{aligned}
$$

Whenever the LTE condition holds, an equation of state relating $\epsilon, p$ and $\rho$ can be obtained, thus closing the system defined by equations (1) to (4). In this work, we have used the equation of state of an ideal relativistic gas, which can be expressed in terms of its enthalpy as:

$$
h=\rho+\frac{\gamma p}{\gamma-1},
$$

with $\gamma=4 / 3$. Considering the contributions of all the involved particles to the total density, mass and energy, we have verified that equation (5) holds in the full range of parameters used in our simulations, with a value of $\gamma$ that deviates from $4 / 3$ with a maximum error of $0.2 \%$. The details of this calculation can be found in [6].

To integrate the above-defined system of equations, we have used the one-dimensional RHD module included in the PLUTO code [9]. The code works by making use of Godunov-type Riemann solvers, of which we have chosen an extension of the HLLC scheme to the equations of RHD (see [10] for the complete details). Among the possible configurations included in PLUTO, we have used a second-order total variation diminishing scheme for spatial reconstruction, and second-order Runge Kutta integration for time evolution. On each time step, the grid was updated in order to better follow total energy density gradients, by means of an adaptive mesh refinement algorithm provided by the CHOMBO library [11].

\section{Simulations}

\subsection{Low density directions}

As a verification of this scheme's applicability to our system, we have performed similar simulations to those in [7], which account for the evolution of the plasma in the lower density regions (see Fig. 1). To be more precise about this, we define the baryon load as $B=M_{B} c^{2} / E_{e^{+} e^{-}}$, where $M_{B}$ is the total integrated mass considering the assumed spherically symmetric distribution, and $E_{e^{+} e^{-}}$is the initial internal energy of the plasma. Namely, $B$ works as an indicator of the mass-to-energy ratio along each direction.

In this part, we will only consider density distributions such that $B<10^{-2}$. As it is explained in detail in [7], in that case the plasma forms a slab that expands while 


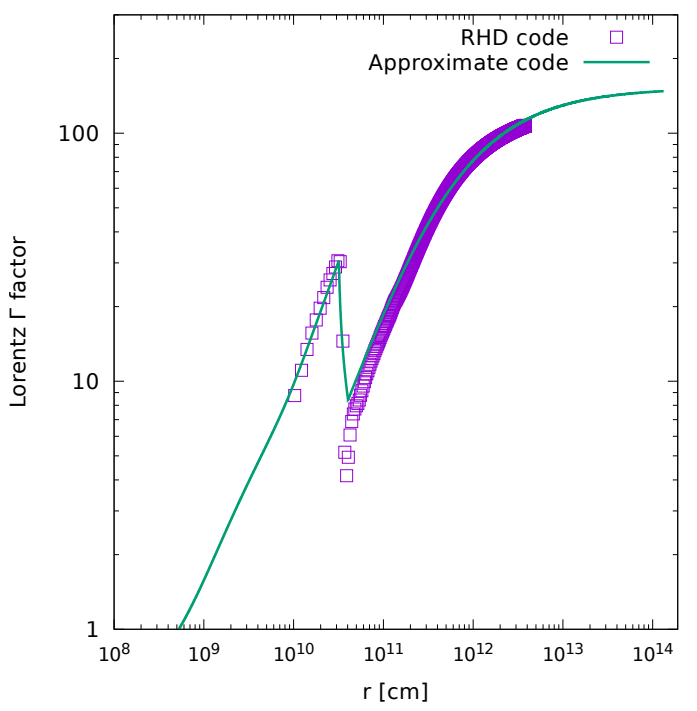

Figure 2. Lorentz factor $\Gamma$ computed in with the PLUTO code, compared with the one computed with the semi-analytic approximate code. For this plot, the values $E_{e^{+} e^{-}}=1.0 \times 10^{53} \mathrm{erg}$ and $B=6.61 \times 10^{-3}$ have been chosen. Similar good agreement is found for other values of $E_{e^{+} e^{-}}$and $B$ as long as $B<10^{-2}$. Reproduced from [6].

accelerating, until it reaches a constant Lorentz factor of roughly $\Gamma \sim 1 / B$. During its evolution, and still under the condition $B<10^{-2}$, the slab's width remains constant when measured in the laboratory frame. This process is studied in [7] using an approximate semi-analytic code that assumes this feature, and allows to predict average intensive quantities by means of conservation equations.

In Fig. 2 we show the comparison between the Lorentz factors computed with both the semi-analytic code and PLUTO, for one particular value of $E_{e^{+} e^{-}}$and $B$. The simulations run with PLUTO show the formation of a slab of constant width, that accelerates accordingly to what is expected from the semi-analytic code. This is in turn consistent with the treatment done in [7], and therefore, the analysis done so far for the prompt emission remains unchanged.

\subsection{High density directions}

Having applied the current scheme to the already-known region of the parameter space, we proceed to study the case $B>10^{-2}$, which corresponds to the interaction of the $e^{+} e^{-}$plasma with the $\mathrm{SN}$ remnant along the higher density directions, and the subsequent emission of the flares. To this end, we have chosen for each simulation an initial density profile that matches the ones obtained in [8] (see Fig. 1). Hence, all the considered profiles were set in the following way:

$$
\rho \propto\left(R_{0}-r\right)^{\alpha}
$$

where $R_{0}$ and $\alpha$, with $2<\alpha<3$, are fitting parameters. Each profile of this kind has a single baryon load, that corresponds to the evolution of the fluid along one particular direction. Similarly, we have taken the velocity to depend

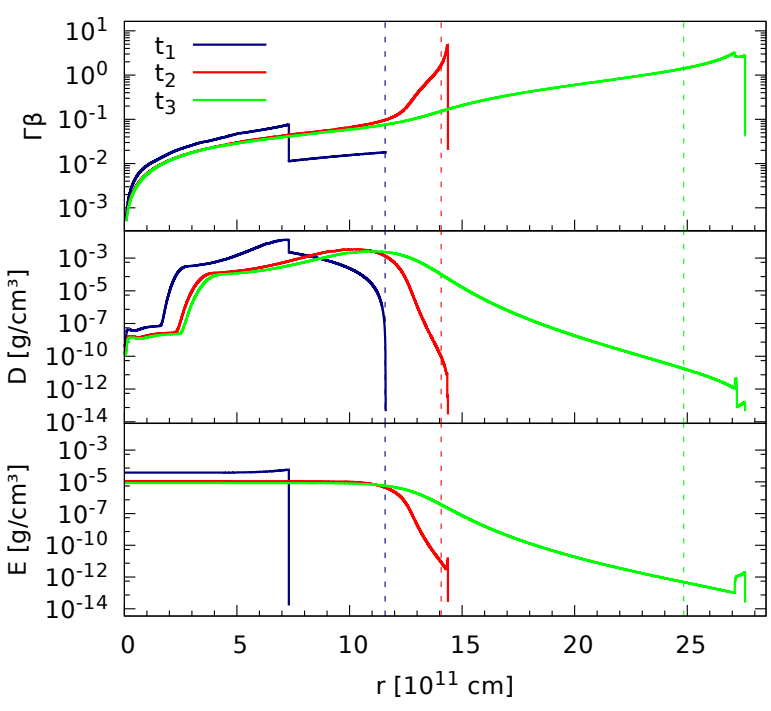

Figure 3. Profiles of $\Gamma v / c$ (up), $D=\rho \Gamma$ (center) and plasma internal energy $\mathcal{E}$ (down) for $B=200$ at three different times, labeled as $t_{1}$ (before the breakout), $t_{2}$ (at the breakout) and $t_{3}$ (after the breakout). The factor $\Gamma v / c$ is approximately equal to $v / c$ when $v \ll c$, and to $\Gamma$ when $v \sim c$. Contributions to $\mathcal{E}$ due to the density have been neglected, in order to substract the baryon's kinetic energy, and show the position of the shock. The vertical dashed lines indicate the position of $R_{p h}$ at each time. See also [6].

on the radius as $v_{r} \propto r$, in order to set an initial profile that corresponds to the homologous expansion of the SN ejecta. Lastly, the plasma is initially contained within a radius of order $10^{8}-10^{9} \mathrm{~cm}$, and has an uniform energy density.

Once the system is let to evolve from these initial conditions, the plasma expands and forms a shock that reaches the outermost part of the SN ejecta. Instead of forming a thin shell with an almost uniform Lorentz factor, the plasma evolves in such a way that the shock is followed by smooth energy and velocity distributions, where the module of the last may differ in several orders of magnitude from one point to another, as it is shown in Fig. 3.

After an initial expansion where $\Gamma$ may reach values of several tenths, the shock rapidly elgulfs enough mass to decelerate and reach a non relativistic velocity distribution, typically in $t<1 \mathrm{~s}$. This is maintained through the whole time the plasma is contained within the SN ejecta, until the breakout, in which the sudden decrease of the density causes the closest areas to the shock to reach relativistic velocities, as it can be seen in Fig. 3. From then on, the matter pushed by the shock -and no longer in homologous expansion- keeps expanding while cooling down.

As the shock propagates inside of the SN ejecta, photons are trapped inside the region occupied by the plasma, since their diffusion timescale is much longer than the dynamical times. Since characteristic equilibrium times are much shorter than both scales (see e.g. [12]), LTE is maintained during the whole evolution of the plasma within the $\mathrm{SN}$ material. However, at the breakout, the plasma reaches 


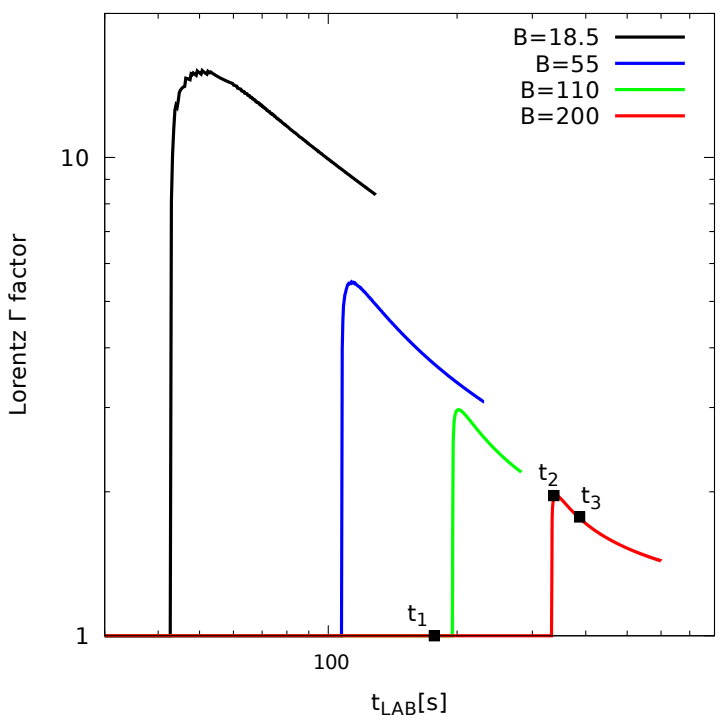

Figure 4. Lorentz gamma at the photospheric radius, calculated for different values of the baryon load, corresponding to different considered directions along the SN ejecta. The three times considered for $B=200$ in Fig. 3 are indicated. Reproduced from [6].

the photosphere, which is an optically thin area located in the outermost regions of the SN ejecta. When this happens, photons begin to escape, and produce what is later observed as a flare.

To perform a comparison between these results and the observed $\Gamma$ factors, we firstly recall from [13] that, before reaching an external observer, most of the photons are lastly scattered from a region peaked at $\tau \sim 1$, where $\tau$ is the optical depth calculated from the observer's line of sight. We hence define the transparency -or photosphericradius $R_{p h}$ as the value of the $r$ coordinate that satisfies $\tau\left(R_{p h}\right)=1$. Finally, we can give an estimation of the observed $\Gamma$ by computing its value at $r=R_{p h}$.

Since $\tau$ is a Lorentz invariant when the total photon cross section is constant, which we assume as a first approximation, it can be calculated in laboratory coordinates as

$$
\tau=\int_{R_{p h}}^{\infty} \mathrm{d} r \sigma_{T} n_{e^{-}}(r)
$$

where we set the cross section to $\sigma_{T}=6.65 \times 10^{-25}$ $\mathrm{cm}^{2}$, i.e., the one corresponding to Thomson scattering by electrons. Moreover, we compute the electron density as $n_{e^{-}}=\rho \Gamma / m_{P}$, where $m_{P}$ is the proton mass. In doing so, we neglect the mass of the electrons, and we assume to have an average of one electron per nucleon.

Fig. 4, already shown in [6], shows the time evolution of $\Gamma\left(R_{p h}\right)$, calculated for four different baryon loads that correspond to four different high-density directions along the SN ejecta. For a high enough $B$, it can be seen that, indeed, $\Gamma\left(R_{p h}\right) \lesssim 4$.

\section{Final remarks and future work}

The performed simulations of the evolution of an $e^{+} e^{-}$ plasma inside of a SN ejecta show as a result the formation, expansion and breakout of a shock. Within the IGC model's parameters, the Lorentz factor at the photospheric radius verifies $\Gamma \lesssim 4$ for a high enough integrated mass across the observer's line of sight. This is consistent with the existence of a thermal emitter expanding at such a Lorentz factor, as it is inferred from the thermal component observed in the X-ray flares.

We have said that RHD holds as long as LTE is granted. However, this is not the case for the thin region close to the shock, from where photons can escape. If this is taken into account, the pressure radiation, which is the dominant one, should be actually smaller, and consequently we should expect the actual Lorentz factors to be even smaller. Therefore, the results of this work must be taken as a superior limit for $\Gamma$. To take this effect into account, we are currently working on a scheme that evolves radiation and massive particles separately, which would allow to compute the emitted luminosity as well.

\section{References}

[1] Rueda, J. A., \& Ruffini, R., ApJL, 758, L7 (2012)

[2] Izzo, L., Rueda, J. A., \& Ruffini, R., A\&A, 548, L5 (2012)

[3] Fryer, C. L., Rueda, J. A., \& Ruffini, R., ApJL, 793, L36 (2014)

[4] Damour, T., Ruffini, R., APS, 35, 7, 463 (1975)

[5] Ruffini, R., Rueda, J. A., Muccino, M., et al., 832, 136 (2016)

[6] Ruffini, R., Wang Y. et al, https://arxiv.org/abs/1704.03821, submitted to ApJ (2017)

[7] Ruffini, R., Salmonson, J. D., Wilson, J. R., Xue, S., A\&A, 359, 855 (2000)

[8] Becerra L., Bianco C. L., Fryer C. L., Rueda J. A., Ruffini R., ApJ 833, 107 (2016)

[9] Mignone, A., Zanni, C., Tzeferacos, P., et al., The Astrophysical Journal Supplement Series, 198, 7 (2011)

[10] Mignone, A., Bodo, G., Mon. Not. R. Astron. Soc., 364, 126 (2005)

[11] Colella, P., Graves, D. T., Johnson, J. N., et al., Chombo software package for AMR applications design document, Tech. Rep. LBNL-6615E (2003)

[12] Aksenov A. G. , Ruffini R., Vereshchagin G. V., Phys. Rev. D 79, 043008 (2009)

[13] Pe'er A., ApJ, 682, 463 (2008) 